\title{
管内电缆导体结构模拟设计优化模型
}

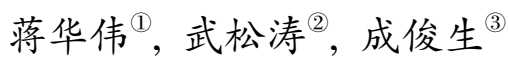 \\ (1) 河南工业大学信息科学与工程学院, 郑州 450001; \\ (2) 中国科学院等离子体物理研究所, 合肥 230031 ; \\ (3) 中国科学院电工研究所, 北京 100080 \\ E-mail: 1hwcad@sohu.com
}

2010-08-08 收稿, 2011-01-10 接受

科技部国际科技合作计划重点项目(2004CB720704)和河南省高等学校青年骨干教师计划(2010GGJS-088)资助

\begin{abstract}
摘要 针对管内电缆导体(Cable-in-Conduit Conductor, CICC)设计中的复杂性问题, 提出了基于 稳定性和应变作用的导体模拟设计模型, 研究了应变对临界电流密度影响的量化效果, 建立了 耦合损耗等多变量制约的超导导体结构设计数学规划方法, 获得优化后的合理导体结构. 并将 数值模拟设计的 CICC 导体与 KSTAR(Korean Superconducting Tokamak Advanced Research)工程 设计进行了比较和分析, 结果显示二者吻合较好.

关键词

CICC

应变

数值模拟设计

稳定性

优化模型
\end{abstract}

CICC 导体即铠装电缆导体, 是由内冷超导导体 (internally-cooled superconductor)演变而来. 早在 1975 年 前它已经引起了研究者的关注 ${ }^{[1]}$, 后来 Chester 提出了 改善导体性能的建议并由 Altov等设计了几种导体结 构 ${ }^{[1 \sim 3]}$, 在这些研究工作基础上, Hoenig 等提出了 CIC(Cable in Conduit)和 CICC 概念模型 ${ }^{[1 \sim 3]}$. 这些大量 探索实验使得 CICC 导体具有超临界氦良好冷却、高电 压绝缘、低交流损耗和多级变位绞缆等优点, 而成为中 国大科学工程 EAST(Experimental Advanced Superconducting Tokamak)、韩国 KSTAR(Korean Superconducting Tokamak Advanced Research)以及国际热 核聚变试验堆 ITER(International Thermal-nuclear Experimental Reactor)上的 TF(Toroidal Field), PF (Poloidal Field), CS(Central Solenoid)和 CC (Correction Coils)的 首选导体.

CICC 导体是由超导组分、稳定体、冷却介质和 导管构成的复合体, 其间的热交换是一个复杂的过程, 加上 CICC 导体的稳定性还受到能量裕度和交流损耗 等的制约, 使得 CICC 导体的设计理论非常复杂, 为 了在理论研究和工程实际中快速和准确地设计 CICC
导体, 研究人员对导体开展了数值模拟设计研究.

起初进行的是导体结构数值设计和失超模拟研 究. 如文献[4]中的 CID(Cable Interactive Designer)就 是用于模拟绞缆的, 它能生成超导电缆的几何形状. 文献[5]中提出子缆包绕及中心冷却的 CICC 导体三 维绞缆数值模拟方法, 它可以输出股线的空间结构、 每级子缆的面积等. 文献[6]中用于模拟迫流冷却导 体失超及稳定性分析的 Gandalf 程序, 它能根据 $\mathrm{CICC}$ 导体的结构、运行条件以及初始热扰动和样品 长度, 来模拟导体失超情况. 另外 Bottura 还在文献[7] 中对聚变实验堆超导磁体设计中稳定性裕度、交流脉 冲(损耗和电流分布)与失超保护(热点温度和最大失 超压力 3 个限制参数进行了研究, 通过择中构建 CICC 超导电缆的稳定性和电流函数, 提出基于简化 稳定性和热点的 CICC 导体有效设计规则. 这些工作 中 CID 等不是根据能量裕度、温度裕度及电流等条 件来计算获得导体结构, 而是通过机械式简单堆积 构成导体的结构; Bottura 的 Gandalf 等主要是针对已 有的 CICC 导体进行稳定性和失超模拟分析, 不涉及 导体结构的模拟设计. 
为获得大电流和快变磁场中的高磁场强度, 高 温超导材料被尝试运用 ${ }^{[8]}$, 但总体上它们还处于研究 阶段, 许多科学问题还有待进一步探索, 目前在低温 铌钛 $(\mathrm{NbTi})$ 超导技术基础上开展了铌三锡 $\left(\mathrm{Nb}_{3} \mathrm{Sn}\right)$ 导 体的研制. 但 $\mathrm{Nb}_{3} \mathrm{Sn}$ 在绕制和热处理线圈时带来的 应变使其难以满足工程应用的需求, 即由热、机械及 其他原因导致的综合应变使 CICC 导体中的丝、股性 能恶化, 从而导致 CICC 导体稳定性进一步降低, 因 此, 应变成为影响着 CICC 导体稳定运行的一个关键 原因. 针对这些问题已进行了相关的理论探索. 文 献[9]精确描述了临界电流密度对不同应变的敏感性, 并分析了临界电流密度的恶化情况和应变对 $n$ 值的 作用. 文献[10,11]中 Zhai 针对热应变和弯绕应变使 ITER 和混合强磁场的 $\mathrm{Nb}_{3} \mathrm{Sn}$ 型 CICC 性能恶化的问 题, 通过周期性载荷试验, 提出高性能的 FEMCAM (Florida Electro-Mechanical Cable Model)模型, 获得 弯应变小于 $1 \%$ 区间的 $n$ 值和临界电流等的恶化情况. 文献[12]中指出 $\mathrm{Nb}_{3} \mathrm{Sn}$ 的脆性是由弯绕和热处理形成 的, 其临界电流密度、上临界磁场、临界温度及 $n$ 值 受应变的影响. 上述工作主要通过实验和模拟手段 获得应变对 $\mathrm{Nb}_{3} \mathrm{Sn}$ 型 $\mathrm{CICC}$ 导体 $n$ 值和临界电流等的 恶化影响或某种关系, 却没有把这些研究结果通过 反向推理用于导体结构的数值模拟设计.

尽管文献[13]根据应变对临界电流密度作用量 化关系进行了导体结构模拟设计，获得多种导体结 构, 但由于在按层(级)数进行模拟设计时, 没有对每 层绞缆根数进行合理性判断, 结果会出现前级根数 远大于后级根数情况, 在工程结构上不满足机械稳 定的要求，一般不被采用，显然这些研究工作没有给 出更为合理的结构方式(见 3.3 节). 因此, 本文在考虑 应变及交流损耗等对稳定性影响基础上, 设计了股 线根数、耦合损耗时间常数等多变量制约的非线性数 学规划方法, 对参数进行优化, 构建 CICC 导体数值 模拟设计模型, 获得优化后更为合理的导体结构.

\section{CICC 导体稳定性}

\subsection{CICC 导体稳定性零维和一维模型}

对短样 CICC 导体精确计算稳定性裕度时，可忽 略加热区的长度. 在股线温度和液氦温度均匀的假 设基础上, 仅用时间作变量, 以获得在短脉冲和长脉 冲期间的稳定性裕度, 由此得到零维模型平衡式 ${ }^{[14]}$.
该模型能简单地描述在恢复时间范围内的局部能量 平衡.

但对长样有高横向电阻的 CICC 导体, 其电流在 全长范围内重新完全分配是很难实现的, CICC 截面 上的液氦温度梯度使导体的稳定性裕度发生急剧变化, 匀质处理已不再适用. 考虑瞬变过程中导管的热扩散 对稳定性的影响, 由流体知识可得液氦(质量、动量 和能量平衡)压缩流体方程, 这样可建立一维模型系 统 ${ }^{[14]}$. 该模型是在综合考虑各种复杂因素后建立的, 主要用于稳定性裕度的计算, 它能预测多值稳定性 的热感应流. 但需对众多参数进行分析, 因此还要解 决稳定和失超计算量大、速度慢且不易控制等问题.

\subsection{CICC 导体稳定性计算}

CICC 的稳定性是导体设计计算的关键. 在上述 稳定性模型基础上, 考虑导体的稳定性受一定温度 范围内液氦焓差的限制，可以对该温度区间内的热 容进行积分得到焓差值. 数学描述如下:

$$
\Delta H=\frac{A_{\mathrm{he}}}{A_{\mathrm{sc}}} \int_{T_{\mathrm{op}}}^{T_{\mathrm{cs}}} C_{\mathrm{he}} \mathrm{d} T,
$$

其中 $A_{\mathrm{he}}$ 和 $A_{\mathrm{sc}}$ 是液氦和股线的面积, $T_{\mathrm{cs}}$ 和 $T_{\mathrm{op}}$ 是分流 温度和设定运行温度, $C_{\mathrm{he}}$ 是液氦的热容.

根据能量平衡并结合在整个干扰区间内液氦和 股线的情况，可以粗略地估计出在好冷却区间、过渡 区间和差冷却区间的稳定性裕度(见文献[15])。这是 后面稳定性裕度计算的基础.

\section{2 应变与临界电流密度关系模型}

$\mathrm{Nb}_{3} \mathrm{Sn}$ 型 CICC 导体的稳定性不仅受磁场强度、温 度、电流密度分布及股线与磁场角度的影响，还与股 线的应变有关. 在模拟 CICC 导体结构设计时, 需要 对应变与临界电流密度的关系进行量化研究.

\section{1 应变计算模型}

应变主要是指 $\mathrm{Nb}_{3} \mathrm{Sn}$ 受到热、机械等作用的效应. 根据材料力学知识，对于周期性均匀的三维实空间 弹性体, 假设它具有光滑连续的边界, 给定边界受拉 或受压的部分, 剩余部分的位移可以给出, 由此产生 的应变就为形变与受拉(压)部分的百分比, 它是影响 临界电流密度的重要因素.

工程设计和数值模拟中的应变根据经验数据来 调整. 假设作用在 CICC 导管上的机械应变全部传给 
导体的丝股, 则 $\mathrm{Nb}_{3} \mathrm{Sn}$ 的有效应变 $\varepsilon$ 可以写成如下 3 个部分之和:

$$
\varepsilon=\varepsilon_{\mathrm{op}}+\varepsilon_{\mathrm{th}}+\varepsilon_{\mathrm{extra}},
$$

其中 $\varepsilon_{\mathrm{op}}$ 为导体纵向应变 (也称为运行时应变), $\varepsilon_{\mathrm{th}}$ 是零 电流时的热应变, $\varepsilon_{\text {extra }}$ 为其他原因导致的额外应变. 严格意义上 $\varepsilon_{\mathrm{op}}$ 和 $\varepsilon_{\mathrm{extra}}$ 是导体真正的纵向应变, 实际 设计中, $\varepsilon_{\mathrm{extra}}$ 与 $n$ 值是影响导体结构的重要参数, 其 变化与导体及股线的性能有关; 热应变 $\varepsilon_{\mathrm{th}}$ 是从实验 数据中估算出来的.

\section{2 与临界电流密度关系模型}

对于应变与临界电流密度关系的研究, 文献[16] 中回顾和比较了 Ekin, Summers, University of Durham, University of Twente 等的临界电流密度 $J_{\mathrm{c}}$ 参数 表达式, 希望由此获得一种通用的方法, 特别是能够 完全适用于 ITER 磁体系统上的 $\mathrm{Nb}_{3} \mathrm{Sn}$ 导体, 但目前 仍在研究中. 本文利用文献 $[16,17]$ 中的实验以及体 积钉扎力公式, 结合给定的磁场强度和应变势能函 数, 还有 $\mathrm{CICC}$ 导体焓值估计公式, 可获得 $\mathrm{Nb}_{3} \mathrm{Sn}$ 临 界电流密度的经验量化描述 ${ }^{[16,17]}$, 它是 Summers 的 研究结果, 并借鉴 ITER-2008 的描述获得 $J_{\mathrm{c}}$ 参数化 形式, 该描述更适合 $\mathrm{Nb}_{3} \mathrm{Sn}$ 复杂的化合物组分以及 应变范围.

$$
J_{\mathrm{c}}\left(B_{0}, T, \varepsilon\right)=C(\varepsilon)\left(B_{\mathrm{C} 2}(T, \varepsilon)\right)^{-1 / 2}\left(1-t^{2}\right)^{2} b^{-1 / 2}(1-b)^{2}, \text { (3) }
$$

其中 $J_{\mathrm{c}}\left(B_{0}, T, \varepsilon\right)$ 是给定运行条件下的临界电流密度, $B_{0}$ 是运行时的背景场强, $T$ 是运行温度, $\varepsilon$ 是股线中 $\mathrm{Nb}_{3} \mathrm{Sn}$ 超导丝在纵向上的总应变.

$$
\begin{gathered}
C(\varepsilon)=C_{0}\left(1-a_{0}|\varepsilon|^{1.7}\right)^{1 / 2}, \\
B_{\mathrm{C} 2}(T, \varepsilon)=B_{\mathrm{C} 20}(\varepsilon)(1-t T)(1-t / 3), \\
b=B_{0} / B_{\mathrm{C} 2}(T, \varepsilon), \\
t=T / T_{\mathrm{C} 0}(\varepsilon), \\
T_{\mathrm{C} 0}(\varepsilon)=T_{\mathrm{C} 0 \mathrm{M}}\left(1-a_{0}|\varepsilon|^{1.7}\right)^{1 / 3}, \\
B_{\mathrm{C} 20}(\varepsilon)=B_{\mathrm{C} 20 \mathrm{M}}\left(1-a_{0}|\varepsilon|^{1.7}\right),
\end{gathered}
$$

其中常数 $a_{0}$ 的取值情况如下: 当 $\varepsilon<0$ 时 ( $\mathrm{Nb}_{3} \mathrm{Sn}$ 受压), $a_{0}=900$; 当 $\varepsilon>0$ 时 $\left(\mathrm{Nb}_{3} \mathrm{Sn}\right.$ 受拉 $), a_{0}=1250 C_{0}, T_{\mathrm{C} 0 \mathrm{M}}$ 和 $B_{\mathrm{C} 20 \mathrm{M}}$ 是根据不同条件由实验获得的经验值.

\section{CICC 导体模拟设计模型}

\subsection{CICC 导体模拟设计思路和假定条件}

CICC 导体设计的基本思路是: 在给定运行条件 下, 根据空隙率、稳定性裕度、温度裕度等参数, 结
合 Stekly 参数 ${ }^{[18]}$ (Stekly 参数作为 CICC 导体全稳定 性判据, 主要是指导体产生的焦耳热及其他扰动能 量与液氦传递热量之间的比率，即单位时间内导体 的干扰与液氦冷却能力之比, 它通过大的铜超比或 冷却周长/横截面来实现)的取值, 利用导体中铜超面 积、湿(润)边周长和电缆空间电流密度及应变影响的 临界电流密度等来推导包括股线根数、超导股线直径 和铜超比等未知参数的矩阵方程, 由此模拟获得 CICC 导体的结构.

在仿真计算中采用了以下假定条件:

(1) 一级子缆采用 3 根股线绞缆, 且都为超导股线.

(2) 为获得合理的电流密度, 设定导体运行在过 渡区间, 即运行电流大于下极限电流并小于上极限 电流, Stekly 参数不大于 1 , 使得导体产生的焦耳热 小于液氦带走的能量.

(3) 若有铜股线则可以处理成三种情况: 在计算 起稳定作用的铜面积和湿边周长时都包括铜股线的 作用; 仅在计算起稳定作用的铜面积时考虑铜股线 的作用; 仅在计算湿边周长时考虑铜股线的作用.

\section{2 导体结构模拟设算法模型}

根据参考文献[13]导体运行在过渡区 $\left(I_{\mathrm{lim}, \mathrm{low}}<I_{\mathrm{op}}<\right.$ $\left.I_{\mathrm{lim}, \text { up }}\right)$ 的假设, 由稳定性裕度、温度裕度和空间电流 密度等可得如下数学模型:

$$
\begin{gathered}
\frac{N_{\mathrm{sc}}^{2} d_{\mathrm{sc}}^{3} R_{\mathrm{cu}}}{R_{\mathrm{cu}}+1}=\frac{4 I_{\mathrm{op}}^{2} \rho_{\mathrm{cu}}}{\pi^{2} \mathrm{~K}_{\mathrm{p}} \alpha h\left(T_{\mathrm{c}}-T_{\mathrm{op}}\right)}=A, \\
R_{\mathrm{cu}}\left(R_{\mathrm{cu}}+1\right)=\frac{\rho_{\mathrm{cu}} \Delta E_{\mathrm{tr}} J_{\mathrm{c}}^{2}}{4 K_{\mathrm{p}} \mathrm{hC} C_{\mathrm{he}}\left(T_{\mathrm{c}}-T_{\mathrm{op}}\right)^{2}} \frac{2 f_{\mathrm{v}}-1}{f_{\mathrm{v}}} d_{\mathrm{sc}}=B d_{\mathrm{sc}}, \\
\frac{R_{\mathrm{cu}}+1}{N_{\mathrm{sc}} d_{\mathrm{sc}}^{2}}=\frac{\pi J_{\mathrm{c}}}{4 I_{\mathrm{op}}}\left(1-\frac{\Delta T_{\mathrm{cs}}}{T_{\mathrm{c}}-T_{\mathrm{op}}}\right)=C,
\end{gathered}
$$

其中 $N_{\mathrm{sc}}, d_{\mathrm{sc}}$ 分别为导体中超导股线根数和直径, $R_{\mathrm{cu}}$ 是铜超比, $I_{\mathrm{op}}$ 是运行电流, $p_{\mathrm{cu}}$ 是铜电阻率, $\pi$ 为圆周率, $K_{\mathrm{p}}$ 是计算湿边周长因子, $\alpha$ 是 Stekly 参数, $h$ 为传热系 数, $T_{\mathrm{c}}$ 是临界温度, $\Delta E_{\mathrm{tr}}$ 是过渡区的稳定性裕度 (见 $1.2), f_{\mathrm{v}}$ 为空隙率, $\Delta T_{\mathrm{cs}}$ 为温度裕度, $J_{\mathrm{c}}$ 为临界电流密度 (计算见前面 2.2 的公式(3), 是综合考虑应变的结果).

\subsection{CICC 导体数值模拟设计}

有了上述假设条件和给定的基本数据及基于应 变的临界电流密度式(3), 利用(10) (12)式, 模拟程 序就可开始导体设计计算. 3 个包含 $R_{\mathrm{cu}}, d_{\mathrm{sc}}, N_{\mathrm{sc}}$ 未知 
量的矩阵方程一般是不难求解的，但当把(12)代人到 (10)时会得到类似于 (11)式的方程, 这说明在空间电 流密度上对 CICC 导体铜组分的优化不是孤立的, 是 与稳定性裕度、温度裕度及临界电流有着必然的联系. 这样实际上就只剩下两个独立方程, 因此无法唯一 确定上面的 3 个未知量; 鉴于此, 可以把 $R_{\mathrm{cu}}, d_{\mathrm{sc}}$ 表示 成 $N_{\mathrm{sc}}$ 的函数, 在数值模拟时, 可以逐步尝试用一、 二、三、四等层的结构(绞缆级数)来确定超导股数 $N_{\mathrm{sc}}$, 再求出符合实际要求的 $d_{\mathrm{sc}}$ 及 $R_{\mathrm{cu}}$.

由(12)得出 $R_{\mathrm{cu}}$ 表达式如下:

$$
R_{\mathrm{cu}}=C N_{\mathrm{sc}} d_{\mathrm{sc}}^{2}-1 .
$$

把(13)代人(10)得出关于 $d_{\mathrm{sc}}$ 的三次方程.

$$
d_{\mathrm{sc}}^{3}-\frac{1}{N_{\mathrm{sc}} C} d_{\mathrm{sc}}-\frac{A}{N_{\mathrm{sc}}}=0 .
$$

根据 CICC 导体结构, 并结合细分超导丝和有利 于分流原则获得合理的股线直径 $d_{\mathrm{sc}}$, 由(13) 获得 $R_{\mathrm{cu}}$.

给定模拟运行条件: $B_{\mathrm{max}}=7.2 \mathrm{~T}, T_{\mathrm{op}}=4.5 \mathrm{~K}, I_{\mathrm{op}}=$ $35200 \mathrm{~A}, \Delta T_{\mathrm{cs}} \geqslant 1 \mathrm{~K}, \mathrm{~d} B / \mathrm{d} t=11 \mathrm{~T} / \mathrm{s}, \Delta E_{\mathrm{tr}}=400 \mathrm{~mJ} / \mathrm{cm}^{3}$. 根 据运行条件和理论计算, 由模拟设计获得 CICC 导体 的股线总根数为 486 , 它包括四级绞缆和五级绞缆两 种情况. 具有四级绞缆的导体结构情况见表 1 , 有 6 种 绞缆方式; 具有五级绞缆的导体结构情况见表 2 , 有 4 种绞览方式.

由表 1 和 2 可知, 该模拟设计并不对每层绞缆根 数进行相对合理性判断, 将获得 $3 \mathrm{SC} \times 3 \times 6 \times 9$ 和 $3 \mathrm{SC} \times$ $6 \times 9 \times 3$ 等多种结构, 使模拟设计的 CICC 结构不唯一, 出现如 $3 \mathrm{SC} \times 6 \times 9 \times 3$ 这样三层(级)根数 9 远大于四层 (级)根数 3 的情况, 它不满足工程结构机械稳定要求, 因此需要进行优化给出更为合理的导体结构.

表 1 四级绞缆结构

\begin{tabular}{ccc}
\hline 二级子缆结构 & 三级子缆结构 & 四级子缆结构 \\
\hline 3 & 6 & 9 \\
3 & 9 & 6 \\
6 & 3 & 9 \\
9 & 3 & 6 \\
6 & 9 & 3 \\
9 & 6 & 3 \\
\hline
\end{tabular}

表 2 五级绞缆结构

\begin{tabular}{cccc}
\hline 二级子缆结构 & 三级子缆结构 & 四级子缆结构 & 五级子缆结构 \\
\hline 3 & 3 & 3 & 6 \\
3 & 3 & 6 & 3 \\
3 & 6 & 3 & 3 \\
6 & 3 & 3 & 3 \\
\hline
\end{tabular}

\section{CICC 导体结构优化}

\section{1 导体结构优化设计}

优化设计的思路和方法：首先是对每层绞缆根 数进行合理性判断, 即满足工程结构上的机械稳定 要求; 然后对符合该条件的导体结构进行交流损耗 判断，通过计算耦合损耗时间常数，获得耦合损耗， 循环计算找出交流损耗(耦合和磁滞损耗)的最小值, 得到优化后的导体结构.

这里主要对 $\mathrm{CICC}$ 导体的每层股线根数和交流损 耗参数等进行优化, 根据一般的优化问题, 只要满足 如下规划条件即可.

$$
\left\{\begin{array}{l}
\min \left(E_{\mathrm{c}}\right)=\min (W+P) \\
\text { s.t. } \quad n_{i} \leqslant n_{j} \quad(i<j)
\end{array}\right.
$$

其中, $n_{i}$ 表示第 $i$ 子缆结构, 即子缆(股线)数目; $E_{\mathrm{c}}$ 为 交流损耗，它包括磁滞损耗 $W($ 值较小，不是关键影 响参数)和耦合损耗 $P$.

由每层股线根数条件可以判断表 1 和表 2 中的 $3 \mathrm{SC} \times 3 \times 6 \times 9$ 和 $3 \mathrm{SC} \times 3 \times 3 \times 3 \times 6$ 结构符合条件.

下面从耦合损耗的计算方法上来探讨上述 CICC 导体结构的选优. 考虑线圈励磁、等离子体放电或破 灭等极端情况，CICC 股线的超导丝大都处在饱和状 态. 根据饱和系数的大小，耦合损耗可采用如下方 法 ${ }^{[19]}$

$$
P= \begin{cases}\frac{2 \theta}{\mathrm{u}_{0}} \dot{B}_{\mathrm{T}}^{2}(1-\beta) & \beta<0.31, \\ \frac{4}{3 \pi} \frac{B_{\mathrm{P}}}{\mathrm{u}_{0}} \dot{B}_{\mathrm{T}} & \beta \geqslant 0.31,\end{cases}
$$

其中 $B_{\mathrm{T}}$ 为横向外场, 饱和系数 $\beta$ 为外部磁场变化率 与穿透场强 $B_{\mathrm{P}}$ 之比, $u_{0}$ 为真空磁导率.

由上可知，耦合损耗时间常数为关键计算参数, 耦合损耗时间常数 $\theta$ 可用下面的公式计算：

$$
\theta=\sum_{n=1}^{\mathrm{N}} \theta_{n},
$$

$\theta_{n}$ 对应于第 $n$ 级绞缆时耦合损耗时间的增加值, 表 达式如下:

$$
\theta_{n}=\frac{\mu_{0}}{2 \rho_{n}}\left(\frac{P_{n}^{*}}{2 \pi}\right)^{2} \frac{1}{1-f_{\mathrm{v} n-1}},
$$

式中 $P_{n}^{*}, \rho_{n}, f_{\mathrm{v} n}$ 分别是 $n$ 级电缆的有效扭距长度、有 效电阻率和空隙率.

有效扭距长度和有效电阻率表达式如下: 


$$
\begin{gathered}
P_{n}^{*}=P_{n}-\frac{r_{n-1}}{R_{n-1}} P_{n-1}, \\
\rho_{n}=\frac{\rho_{\mathrm{b}} \mathrm{e}_{\mathrm{b}}}{\varepsilon_{n} R_{n-1}},
\end{gathered}
$$

其中 $P_{n}, R_{n}, r_{n}$ 和 $\varepsilon_{n}$ 分别是 $n$ 级电缆似在扭距长度、外 半径、扭绞半径及接触面比率, $\rho_{\mathrm{b}} e_{\mathrm{b}}$ 是接触电阻层的 表征. 当 $n=2$ 时, $r_{1}$ 是股线中超导丝的半径, $R_{1}$ 是股线 的半径.

通过模拟计算可获得上述两种 CICC 导体结构的 耦合损耗时间常数和交流损耗(见表 3). 取交流损耗 值较小者, 由此得出 $3 \mathrm{SC} \times 3 \times 3 \times 3 \times 6$ 的结构更为合理.

模拟优化后获得的 CICC 结构见图 1. 由图可知: 它的一级子缆是由 3 根 $\mathrm{Nb}_{3} \mathrm{Sn}$ 超导股线构成, 二级子 缆由 3 根一级子缆构成, 三级子缆由 3 根二级子缆构 成, 四级子缆由 3 根三级子缆构成, 五级子缆由 6 根 四级子缆构成, 最后穿管, 并挤压形成 CICC 导体结 构.

\section{2 结果比较分析}

由于韩国 KSTAR 磁体系统的 TF 及 1-5PF 线圈的 CICC 导体采用了 $\mathrm{Nb}_{3} \mathrm{Sn}$ 材料. 由数据库中调出相应运 行条件下的数据, 将模拟优化结果与 KSTAR 的第一 五的 PF 的 CICC 导体设计值比较如表 4 和 5 .

表 3 导体参数计算

\begin{tabular}{ccc}
\hline 参数 & $3 \mathrm{SC} \times 3 \times 3 \times 3 \times 6$ & $3 \mathrm{SC} \times 3 \times 6 \times 9$ \\
\hline$\theta_{0}(\mathrm{~ms})$ & 0.8677 & 0.8677 \\
$\theta_{1}(\mathrm{~ms})$ & 1.3894 & 1.3894 \\
$\theta_{2}(\mathrm{~ms})$ & 3.6906 & 3.6906 \\
$\theta_{3}(\mathrm{~ms})$ & 8.1790 & 13.209 \\
$\theta_{4}(\mathrm{~ms})$ & 12.389 & 27.735 \\
$\theta_{5}(\mathrm{~ms})$ & 18.095 & $/$ \\
$\theta_{t}(\mathrm{~ms})$ & 44.611 & 46.892 \\
$E_{\mathrm{c}}\left(\mathrm{mJ} / \mathrm{cm}^{3}\right)$ & 203.24 & 217.37 \\
\hline
\end{tabular}

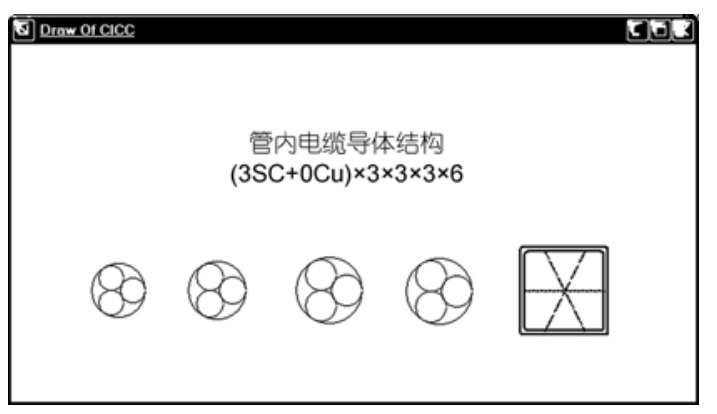

图 1 数值仿真得到的 CICC 导体结构
表 4 工程设计与仿真设计结构结果比较

\begin{tabular}{ccc}
\hline 结构 & 工程设计值 & 数值优化结果 \\
\hline 一级子缆 & $3 \mathrm{SC}$ & $3 \mathrm{SC}$ \\
二级子缆 & $3 \mathrm{SC} \times 3$ & $3 \mathrm{SC} \times 3$ \\
三级子缆 & $3 \mathrm{SC} \times 3 \times 3$ & $3 \mathrm{SC} \times 3 \times 3$ \\
四级子缆 & $3 \mathrm{SC} \times 3 \times 3 \times 3$ & $3 \mathrm{SC} \times 3 \times 3 \times 3$ \\
$\mathrm{CICC}$ 导体 & $3 \mathrm{SC} \times 3 \times 3 \times 3 \times 6$ & $3 \mathrm{SC} \times 3 \times 3 \times 3 \times 6$ \\
\hline
\end{tabular}

表 5 工程设计与数值仿真设计参数比较

\begin{tabular}{ccc}
\hline 参数 & 工程设计值 & 数值优化结果 \\
\hline$A_{\mathrm{cu}}\left(\mathrm{mm}^{2}\right)$ & 170.3 & 167.6 \\
$A_{\mathrm{sc}}\left(\mathrm{mm}^{2}\right)$ & 61.9 & 60.2 \\
$T_{\mathrm{c}}(\mathrm{K})$ & 12 & 12.57 \\
$T_{\mathrm{cs}}(\mathrm{K})$ & $<10.2$ & 6.58 \\
$I_{\mathrm{c}}(\mathrm{A})$ & 80000 & 84488 \\
$d_{\mathrm{sc}}(\mathrm{mm})$ & 0.78 & 0.779 \\
$R_{\mathrm{cu}}$ & 1.5 & 1.51 \\
$\Delta E_{\mathrm{tr}}\left(\mathrm{mJ} / \mathrm{cm}^{3}\right)$ & 400 & 473.6 \\
$E_{\mathrm{c}}\left(\mathrm{mJ} / \mathrm{cm}^{3}\right)$ & $\leqslant 250$ & 203.24 \\
\hline
\end{tabular}

由表 4 可知, 数值仿真给出的 CICC 结构与工程 设计结果相一致, 这是基于稳定性、应变影响以及交 流损耗进行优化设计得到的结果.

由表 5 可得经优化设计获得 CICC 导体的交流损耗 $E_{\mathrm{c}}$ 小于工程设计值, 表明优化获得的扰动小于工程设计 结果; 优化导体的稳定性裕度 $\Delta E_{\mathrm{tr}}$ 大于工程设计值, 显 示优化后导体相对更稳定; 同时优化设计中交流损耗小 于工程设计和模拟设计的稳定性裕度值, 这说明优化后 的 CICC 导体是可以在该条件下很好的稳定运行.

表 5 也显示优化设计与工程设计在参数上有不 同之处. 如在数值仿真中, 由于绞缆系数不同导致 $A_{\mathrm{cu}}$ 的不同, $I_{\mathrm{c}}$ 的不同主要是因为计算方法不同所致(数值 仿真采用 $I_{\mathrm{c}}=A_{\mathrm{sc}} \times J_{\mathrm{c}}$, 工程上是由经验给定某一运行条 件下单股超导线的临界电流计算而得), 稳定性裕度 $\Delta E_{\mathrm{tr}}$ 不同主要与 $A_{\mathrm{cu}}$ 有关. 针对这种问题, 在仿真设计 设计中拟采用合适的湿边周长系数(综合考虑 $\mathrm{CICC}$ 导体结构, 特别是初级子缆、超导股线根数)等来弥 补计算误差.

\section{5 结论}

给出导体稳定性理论概念, 结合 CICC 导体工程 设计经验知识, 研究了 $\mathrm{Nb}_{3} \mathrm{Sn}$ 的应变对临界电流密 度影响的量化模型. 给出了 CICC 导体数值仿真设计 的基本原则和思路, 并利用一级子缆采用 3 根超导股 线、 $\mathrm{CICC}$ 导体运行于过渡区间及纯铜线起不同作用 假定条件, 获得多种 CICC 导体模拟设计结构. 
根据交流损耗及工程上子缆(股线)根数和绞缆 级数确定机理, 建立了每层股线根数和耦合损耗时 间常数等多变量制约的导体结构设计数学规划方法, 对参数进行优化, 通过仿真得出 CICC 导体绞缆情况,
在优化基础上获得合理的导体结构. 比较了数值仿 真设计结果与 KSTAR 工程设计值, 并对误差产生原 因进行了分析, 还给出了改进方法. 这为磁体系统中 $\mathrm{CICC}$ 导体的设计提供了有用的参考.

\section{参考文献}

1 Dresener L. Twenty years of cable-in-conduit conductors: 1975-1995. J Fus Energ, 1995, 14: 3-12

2 Seeber B. Hand book of applied superconductivity. London: Institute of Physics Publication, 1998: 265-280

3 Renard B, Martinez A, Duchateau J L, et al. Transverse heat transfer coefficients on a full size dual channel CICC ITER conductor. Cryogenics, 2006, 46: 530-540

4 Bottura L, Breschi M, Rosso C. Analysis of electrical coupling parameters in superconducting cables. Cryogenics, 2003, 43: 233-239

5 Qin J G, Wu Y. A 3D numerical model study for superconducting cable pattern. Fus Eng Des, 2010, 85: 109-114

6 Marinucci C, Bottura L, Vecsey G, et al. The quell experiment as a validation tool for the numerical code gandalf. Cryogenics, 1998, 38: 467-477

7 Bottura L. Stability, protection and ac loss of cable-in-conduit conductors-a designer's approach. Fus Eng Des, 1993, 20: 351-362

8 马廷灿, 万勇, 姜山. 铁基超导材料制备研究进展. 科学通报, 2009, 54: 557-567

9 Zhang P X, Liang M, Tang X D, et al. Strain influence on $J_{\mathrm{c}}$ behavior of $\mathrm{Nb}_{3}$ Sn multifilamentary strands fabricated by internal tin process for ITER. Physic C, 2008, 46: 1843-1846

10 Zhai Y H, Mark D B. Florida electro-mechanical cable model of $\mathrm{Nb}_{3}$ Sn CICCs for high-field magnet design. Supercond Sci Technol, 2008, 21: 1-15

11 Zhai Y H. Electro-mechanical modeling of $\mathrm{Nb}_{3}$ Sn CICC performance degradation due to strand bending and inter-filament current transfer. Cryogenics, 2010, 50: 149-157

12 Muller $\mathrm{H}$, Schneider $\mathrm{T}$ h. Heat treatment of $\mathrm{Nb}_{3}$ Sn conductors. Cryogenics, 2008, 48: 323-330

13 蒋华伟, 武松涛. 基于应变 CICC 导体模拟设计模型研究. 电子学报, 2010, 38: 1334-1388

14 Bottura L, Luongo C. Superconductors, stability in forced flow. In: John G W, ed. Wiley Encyclopedia of Electrical and Electronic Engineering. Milton: John Wiley \& Sons, 1999. 126-158

15 Jiang H W, Wu S T. Research of simulation design for CICC based on energy margin and temperature margin. IEEE Trans Appl Supercond, 2010, 20: 1436-1439

16 Bottura L, Bordini B. $J_{c}(B, T, \varepsilon)$ parameterization for the ITER $\mathrm{Nb}_{3}$ Sn production.IEEE Trans Appl Supercond, 2009, 19: 1521-1524

17 Summers L T, Guinan M W, Miller J R, et al. A model for the prediction of $\mathrm{Nb}_{3} \mathrm{Sn}$ critical current as a function of field, temperature, strain and radiation damage. IEEE Trans Magn, 1991, 27: 2041-2044

18 Wilson M N. Superconducting Magnets. Oxford: Oxford University Press, 1983: 156--268

19 Fang J, Weng P D, Chen Z M, et al. The ac losses measurement and analysis of superconducting NbTi CICC for HT-7U superconducting Tokamak. Plasm Sci Technol, 2003, 14: 76-82 